\title{
Characterization of shape and dimensional accuracy of incrementally formed titanium sheet parts
}

\author{
Amar Kumar Behera ${ }^{1}$, Bin $\mathrm{Lu}^{2,3}$, and Hengan $\mathrm{Ou}^{1, \mathrm{a}}$ \\ ${ }^{1}$ Department of Mechanical, Materials and Manufacturing Engineering, Faculty of Engineering, \\ University of Nottingham, Nottingham NG7 2RD, UK \\ ${ }^{2}$ Institute of Forming Technology and Equipment, Shanghai Jiao Tong University, Shanghai 200030, \\ China \\ ${ }^{3}$ Department of Mechanical Engineering, University of Sheffield, Sheffield S1 3JD, UK
}

\begin{abstract}
Single Point Incremental Forming (SPIF) is a relatively new process that has been recently used to manufacture medical grade titanium sheets for implant devices. However, one limitation of the SPIF process may be characterized by dimensional inaccuracies of the final part as compared with the original designed part model. Elimination of these inaccuracies is critical to forming medical implants to meet required tolerances. In this study, a set of basic geometric shapes were formed using SPIF to characterize the dimensional inaccuracies of grade 1 titanium sheet parts. Response surface functions are then generated to model the deviations at individual vertices of the STL model of the part as a function of geometric shape parameters such as curvature, depth, wall angle, etc. The generated response functions are further used to predict dimensional deviations in a specific clinical implant case. The predicted deviations show a reasonable match with the actual formed shape and are used to generate optimized tool paths for minimized shape and dimensional inaccuracy. Further, an implant part is then made using the accuracy characterization functions for improved accuracy. The results show an improvement in shape and dimensional accuracy of incrementally formed titanium medical implants.
\end{abstract}

\section{Introduction}

Titanium is the material of choice in Class III medical implants due to its biological inertness, strength, lightweight nature, bio-compatibility and low cost production [1]. Forming titanium into desired implant shapes within a specific time-frame is therefore of fundamental importance to clinical practice. To enable this, one relatively new manufacturing technique that has come forth is Incremental Sheet Forming (ISF). A number of efforts have been made to manufacture implants and supports for different parts of the human body such as the skull [2-5], knee [6], face [7], ankle support [8].

Despite a number of efforts to make medical implant shapes using SPIF, making these parts with high accuracy has been a problem. Specifically, no definite characterization of titanium implants made

\footnotetext{
${ }^{\text {a }}$ Corresponding author: h.ou@nottingham.ac.uk
}

This is an Open Access article distributed under the terms of the Creative Commons Attribution License 4.0, which permits unrestricted use, distribution, and reproduction in any medium, provided the original work is properly cited. 
by SPIF is currently available. Some accuracy characterization techniques are available. These include the use of Multivariate Adaptive Regression Splines (MARS) within a feature based framework for predicting the behavior of simple features and feature interactions [9] and a local geometry matrix to predict springback [10]. However, these works did not provide any methods for predicting inaccuracy in freeform implant shapes. Furthermore, titanium is a material not covered by current accuracy models.

To overcome the limitations of the current accuracy characterization techniques, an effort is made in this work to generate accuracy response surfaces for freeform shapes. This is done by studying the accuracy behavior of ellipsoidal shapes formed using SPIF. The major and minor axes of the ellipsoids are used as parameters in the characterization models generated using MARS. These models are then used to predict accuracy behavior of new implant geometries and the predicted behavior is then used to compensate the parts.

\section{Accuracy characterization methodology}

The accuracy of a part formed by incremental forming is typically determined by measuring the same with metrology tools such as a laser scanner or a coordinate measuring machine. After carrying out this measurement, a point cloud representing the part can be generated and this point cloud can be meshed and then compared with a mesh representing a nominal model obtained from the computer aided design (CAD) model of the part. The measurement process with a point cloud gives the coordinates of the formed part, which is a large data set, often as high as 100,000-500,000 points for a single part. Hence, the deviations with respect to the CAD model, also form a large dataset. These deviations can thus be modeled as function of geometrical parameters for points on the surface of the nominal model. However, to do this, a robust, statistical tool for high dimensional data is needed.

Some tools that are currently available and used in modeling of high dimensional data include Generalized Additive Models (GAM), Multivariate Adaptive Regression Splines (MARS), Minimax Probability Machine Regression (MPMR) and Least Square Support Vector Machine (LSSVM) [11]. Of these, MARS has already been used to generate models of accuracy behavior in planar and ruled features made with specific materials such as AA 3103 and DC01 [12]. This technique was also applied in the current study.

In this study, MARS models are generated as a sum of basis spline functions which are chosen using a forward pass and backward pruning step. The basis functions take one of three forms: a) constant b) hinge functions of the type $\max (0, \mathrm{x}-\mathrm{k})$ or $\max (0, \mathrm{k}-\mathrm{x})$ where $\mathrm{k}$ is constant called the "knot" c) product of hinge functions. To generate the MARS models, accuracy data is linked to geoemterical and process parameters for individual points on the CAD model of the part and then fed to a statistical package "Earth", available within the software "R" [13]. It was important to find out suitable parameters to characterize the accuracy, and this is described in Sect. 2.1.

\subsection{Model parameters}

To build regression models, we need to select geometric and process related variables that potentially affect the accuracy behaviour of the formed part. In prior work for planar and ruled features, it has been shown that the distance to the feature borders affects the accuracy behaviour [13]. In the case of freeform features, the obvious problem is the lack of a generic defining distance in the horizontal plane of the backing plate as freeform surfaces do not have an immediately obvious symmetry and as such a defining distance can be problematic in characterizing the accuracy. However, when we consider the case of a cranial implant, we observe that the shape of the implant can be thought of as being close to that of an ellipsoid (Fig. 1), characterized by a major axis and minor axis. This observation was further supplemented by experimental results, discussed later in Sect. 3, which showed that the accuracy in the direction of the major axis was different from the accuracy in the direction of the minor axis at a specific 


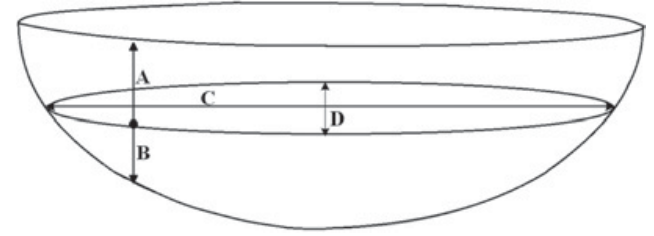

Figure 1. Geometrical model parameters for ellipsoid.

cross-sectional depth. It may also be noted that for ruled features, the maximum principal curvature is a model parameter, while the minimum principal curvature is zero. In freeform surfaces, both principal curvatures have a finite value and hence, both can be included as parameters in the modelling.

The following parameters are thus used to predict the 3D deviation from the CAD model at a point:

1. normalized distance from the point to the bottom of the feature $\left(d_{o}=\mathrm{B} /(\mathrm{A}+\mathrm{B})\right)$,

2. total vertical length of the feature at the vertex $\left(d_{v}=(\mathrm{A}+\mathrm{B})\right.$ in Fig. 1),

3. major axis length on slicing the feature at the depth of the point $\left(l^{\max }=\mathrm{C}\right)$

4. minor axis length on slicing the feature at the depth of the point $\left(l^{\min }=\mathrm{D}\right)$

5. maximum curvature at the point, $k^{\max }$

6. minimum curvature at the point, $k^{\text {min }}$

7. wall angle at the vertex (in radians), $\alpha$

8. angle of the tool movement with respect to the rolling direction of the sheet (in radians), $\omega$.

\subsection{Experimental details}

A hemispherical tool of radius $5 \mathrm{~mm}$ was used for all the tests along with a feedrate of $2 \mathrm{~m} / \mathrm{min}$. A soft low melting-point paste lubricant, Rocol RTD Compound, was applied during the process. Four ellipsoids were used for training the models with major and minor axis diameters $110 \times 60,110 \times 70$, $110 \times 90$ and $90 \times 60$ (all dimensions in $\mathrm{mm}$ ). All parts were made in grade 1 titanium alloy of thickness $0.5 \mathrm{~mm}$. A backing plate with an elliptical cross-section corresponding to the dimensions of the top contour of the part was used for each test. A contouring tool path with constant scallop height of $0.05 \mathrm{~mm}$ was used for forming the parts. The formed parts were unclamped and measured with a $3 \mathrm{D}$ coordinate measuring machine to generate point clouds representing the formed part shape. In the current work, the sheets used are thin and so the deformation on unclamping is significant. Hence, it is important to develop models for the net effect of deviations due to plastic deformation while forming and deviations due to unclamping.

\section{Characterization results}

The accuracies of the formed ellipsoids (shown in Fig. 2) are listed in Table 1. It can be seen that changing the diameters of the major and minor axes affects the accuracy of the part. The smallest part shows the highest over forming. This can be attributed to the low wall angles in the part, which are usually responsible for over forming. The largest part with the highest wall angles (top contour of $110 \mathrm{~mm} \times 90 \mathrm{~mm}$ ) shows exclusively under forming. This is due to two reasons: a) ellipsoids are essentially positive curvature freeform surfaces and positive curvature tends to under form [14], b) the biggest part has high wall angles in the initial forming steps and high wall angles in a positive curvature region are known to under form [14]; this leads to the lower depths also showing under forming being a continuation of the top surface. 


\section{MATEC Web of Conferences}

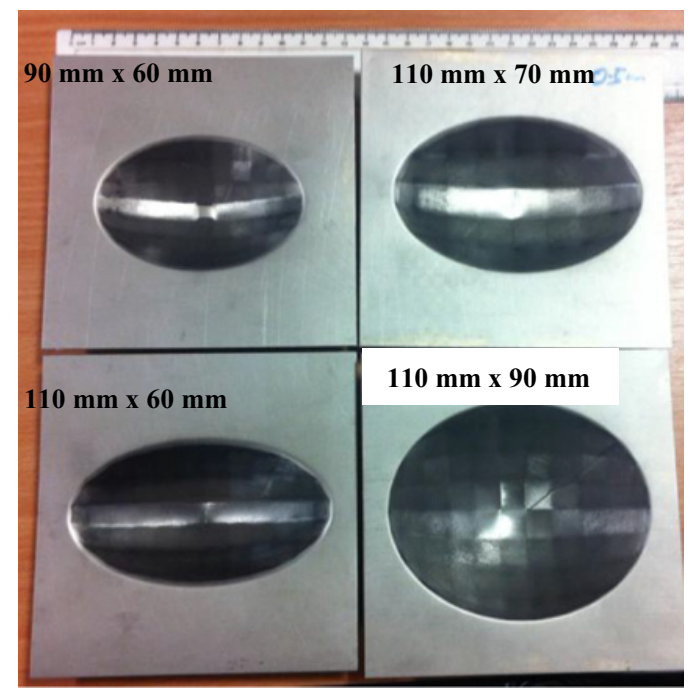

Figure 2. Ellipsoids used for training the MARS models.

Table 1. Accuracies of formed ellipsoids (Negative deviations indicate over forming while positive deviations indicate under forming).

\begin{tabular}{|c|c|c|c|c|c|}
\hline Major axis diameter & Minor axis diameter & Min. deviation & Max. deviation & Mean deviation & Standard deviation \\
\hline 110 & 60 & -0.6 & 2.44 & 0.38 & 0.41 \\
\hline 110 & 70 & -1.16 & 0.95 & 0.16 & 0.38 \\
\hline 110 & 90 & 0 & 1.93 & 0.60 & 0.33 \\
\hline 90 & 60 & -1.39 & 0.64 & -0.34 & 0.27 \\
\hline
\end{tabular}

* All dimensions are in $\mathrm{mm}$.

The MARS model was trained with accuracy data from these tests resulting in the following model.

$$
\begin{aligned}
& e=-0.65+0.35 * \max \left(0,0.97-d_{o}\right)+7.2 * \max \left(0, d_{o}-0.97\right)-0.024 * \max \left(0, d_{v}-45\right) \\
& +0.0049 * \max \left(0,56-d_{v}\right)+0.71 * \max \left(0, d_{v}-56\right)-0.008 * \max \left(0, l^{\max }-97\right) \\
& +0.028 * \max \left(0,17-l^{\text {min }}\right)+0.013 * \max \left(0, l^{\min }-17\right)+3.9 * \max \left(0, k^{\max }+0.0061\right) \\
& +14 * \max \left(0,6.8 * 10^{-5}-k^{\text {min }}\right)+6.3 * \max \left(0, k^{\min }-6.8 * 10^{-5}\right)-1.4 * \max (0,0.62-\alpha) \\
& -1.2 * \max (0, \alpha-0.62)+3.5 * \max (0, \omega-1.2)+0.59 * \max (0,1.3-\omega)-13 * \max (0, \omega-1.3)
\end{aligned}
$$

where, $e=$ deviation at STL vertex and the remaining abbreviations are the same as in Sect. 2.1.

\section{Benchmark validation test cases}

\subsection{Test geometries}

The model generated in Eq. (1) was validated in two steps. First, it was tested against the training sets. Then, it was used to predict a new part, which is a cranial implant. The geometry of the cranial implant is shown in Fig. 3. 

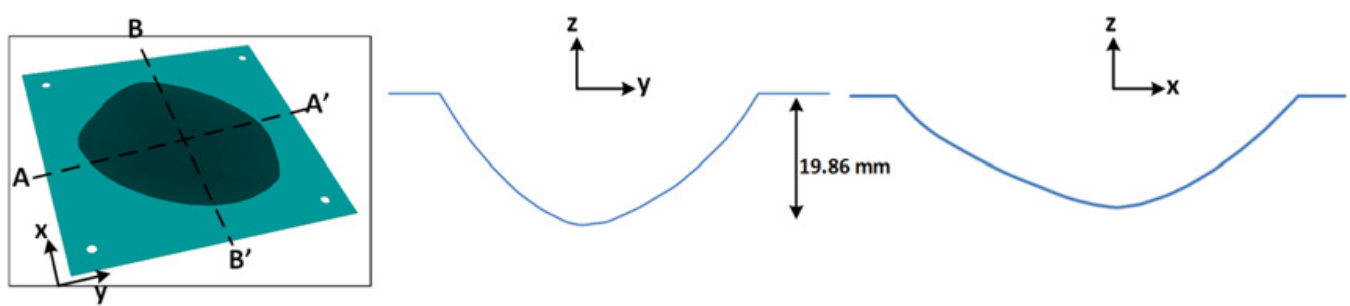

Figure 3. Cranial implant geometry in isometric view, $\mathrm{x}$-section along $\mathrm{AA}^{\prime}$ and $\mathrm{y}$-section along $\mathrm{BB}^{\prime}$.

Table 2. Prediction accuracies of benchmark test cases (All dimensions are in $\mathrm{mm}$ ).

\begin{tabular}{|c|c|c|c|c|}
\hline Major axis diameter & Minor axis diameter & Min deviation & Max deviation & Mean deviation \\
\hline 110 & 60 & -0.42 & 0.32 & 0.02 \\
\hline 110 & 70 & -0.93 & 0.61 & -0.23 \\
\hline 110 & 90 & -0.69 & 0.53 & 0.04 \\
\hline 90 & 60 & -0.89 & 0.95 & -0.01 \\
\hline \multicolumn{2}{|r|}{ Cranial plate } & -1.00 & 1.00 & -0.82 \\
\hline
\end{tabular}

\subsection{Model validation results}

The results of the validation are presented in Table 2. Here, the predicted model using Eq. (1) is compared with the actual formed part from experiments and the prediction error is thereby found. It is seen that the training test cases show a mean deviation close to zero, while the implant shows an error of $-0.82 \mathrm{~mm}$. The error in the prediction of the formed shape of the implant is [-1.0 $\mathrm{mm}, 1.0 \mathrm{~mm}$, which is not as good as the prediction for the ellipsoids. The reason for the slightly poor prediction is possibly that the ellipsoid is still not a perfect representation of the curvatures in the cranial plate and the major axis and minor axis dimensions obtained for the cranial plate are only an approximation of the material deformation in the case of an ellipsoid. However, it would be reasonable to say that the model in Eq. (1) is a good starting point for prediction of positive curvature freeform surfaces and this model can be improved further either by choosing different geometrical parameters for the model or using more training sets.

\subsection{Compensation technique}

The compensation of the parts is carried out by translating individual vertices in the nominal CAD model of the part normal to the part geometry by a magnitude equal to a compensation factor multiplied with the predicted deviation at the point. This follows the strategy outlined by Bambach et al. [15]. Three different compensation factors were tried out, $0.7,1$ and 1.3.

\subsection{Accuracy of compensated implant}

The results of the compensation are presented in Table 3 along with the result for a part made without compensation (compensation factor 0). It can be seen that the part with the best accuracy is realised with a compensation factor of 0.7. A color plot of the part accuracy and that of the uncompensated part is shown in Fig. 4 to illustrate the utility of using the model in Eq. (1) in forming parts with higher accuracy. It can be seen that with increasing compensation, the over forming in the part increases and the under forming decreases. 


\section{MATEC Web of Conferences}

Table 3. Results of forming a cranial implant using MARS model with different compensation factors.

\begin{tabular}{|c|c|c|c|c|}
\hline Compensation factor & Minimum deviation & Maximum deviation & Mean deviation & Standard Deviation \\
\hline 0 & -0.47 & 1.02 & 0.09 & 0.24 \\
\hline 0.7 & -0.57 & 0.53 & 0.05 & 0.19 \\
\hline 1 & -0.75 & 0.36 & -0.06 & 0.17 \\
\hline 1.3 & -0.96 & 0.25 & -0.31 & 0.23 \\
\hline
\end{tabular}

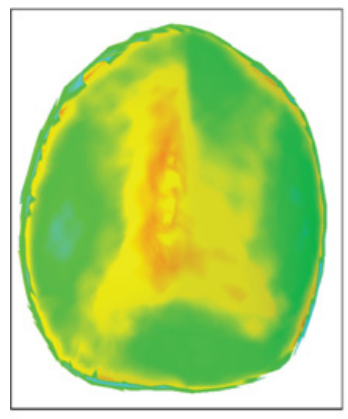

(a)

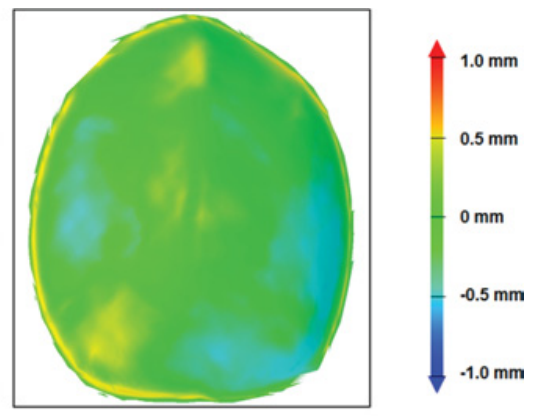

(b)

Figure 4. Accuracy plot of cranial implant manufactured with (a) uncompensated and (b) compensated tool paths.

\section{Conclusions}

In this study, a method to predict the accuracy behaviour of incrementally formed freeform titanium parts was developed. Multivariate Adaptive Regression Splines (MARS) were used to develop a model that predicts the accuracy. The models are based on four training sets formed as ellipsoids. The major and minor axes of the ellipsoid at the depth (z-axis) cross-section of individual points in the part are used as variables in the model. In addition, both maximum and minimum curvatures are used as predictors. The results show that the model is reliable in predicting ellipsoids from the training set, while the prediction accuracy deteriorates for a new part such as a cranial implant. Using the model, part compensation was carried out for a grade 1 titanium implant part and the accuracy of the formed part was seen to improve vis-à-vis the uncompensated part. Further studies can include improving the model with better predictors or a new prediction technique such as GAM, and incorporating a mixed model with curvatures from experiments with ruled surfaces.

This work was supported by the Engineering and Physical Science Research Council of UK (EP/L02084X/1), the Marie Curie International Incoming Fellowship (628,055\&913,055), International Research Staff Exchange Scheme (IRSES, MatProFuture project, 318,968) within the $7^{\text {th }}$ EC Framework Programme (FP7).

\section{References}

[1] S. Aydin, B. Kucukyuruk, B. Abuzayed, S. Aydin, G.Z. Sanus, J Neurosci Rural Pract, 2 (2011) $162-167$

[2] J.R. Duflou, A.K. Behera, H. Vanhove, L.S. Bertol, Key Eng Mater, 549 (2013) 223-230

[3] A. Göttmann, M. Korinth, V. Schäfer, B.T. Araghi, M. Bambach, G. Hirt, Future Trends in Production Engineering, Springer Berlin Heidelberg (2013) pp. 287-295

[4] B. Lu, H. Ou, S.Q. Shi, H. Long, J. Chen, Int J Mater Form (2014) (published online)

[5] B. Lu, D.K. Xu, R.Z. Liu, H. Ou, H. Long, J. Chen, Key Eng Mater, 639 (2015) 535-542 
[6] P.D. Eksteen, A.F. Van der Merwe, Proceedings of the International Conference on Computers \& Industrial Engineering (CIE 42), Cape Town, South Africa (2012) pp. 569-575

[7] R. Araujo, P. Teixeira, L. Montanari, A. Reis, M.B. Silva, P.A. Martins, Prosthetics and orthotics international, 38 (2014) 369-378

[8] G. Ambrogio, L. De Napoli, L. Filice, F. Gagliardi, M. Muzzupappa, J. Mater. Proces. Technol., 162-163 (2005) 156-162

[9] J. Verbert, A.K. Behera, B. Lauwers, J.R. Duflou, Key Eng Mater, 473 (2011) 841-846

[10] M.S. Khan, F. Coenen, C. Dixon, S. El-Salhi, M. Penalva, A. Rivero, Int J Adv Manuf Technol, (2014) 1-12

[11] P. Samui, Journal of Advanced Manufacturing Systems, 13 (2014) 237-246

[12] A.K. Behera, J. Gu, B. Lauwers, J.R. Duflou, Key Eng Mater, 504-506 (2012) 919-924

[13] A.K. Behera, J. Verbert, B. Lauwers, J.R. Duflou, Comput Aided Design, 45 (2013) 575-590

[14] A.K. Behera, PhD Thesis, Katholieke Universiteit Leuven, 2013

[15] G. Hirt, J. Ames, M. Bambach, R. Kopp, Cirp Ann-Manuf Techn, 53 (2004) 203-206 